\title{
Misfortune never comes singly - the first case of COVID-19 with rupture of thoracic aortic aneurysm
}

\author{
Kun Zhao \\ the First Affiliated Hospital of Zhengzhou University \\ Zhang Cao \\ the First Affiliated Hospital of Zhengzhou University \\ Changju Zhu \\ the First Affiliated Hospital of Zhengzhou University \\ Yi Zhang \\ the First Affiliated Hospital of Zhengzhou University

\section{Feifan Chen} \\ the First Affiliated Hospital of Zhengzhou University
}

\section{Case Report}

Keywords: COVID-19, thoracic aortic aneurysm, cytokine storm syndrome

Posted Date: March 25th, 2020

DOI: https://doi.org/10.21203/rs.3.rs-19416/v1

License: (c) (1) This work is licensed under a Creative Commons Attribution 4.0 International License. Read Full License 


\section{Abstract}

Background: SARS-CoV-2 is a newly discovered virus, leading COVID-19 a global threaten nowadays. Case Presentation: The first case of a patient with a thoracic aortic aneurysm (TAA) that became infected with severe acute respiratory syndrome coronavirus 2 (SARS-CoV-2) was reported here. The patient died suddenly from a ruptured aorta $19 \mathrm{~h}$ after hospital admission. Conclusion: COVID-19 patients with TAA should attract the highest vigilance as COVID-19 might expedite the process of TAA rupture through cytokine storm syndrome, leading to rapid death with little or no warning signs.

\section{Background}

Cases of an initially unidentified viral pneumonia were first reported in Wuhan, Hubei Provence, China, in December 2019. A novel coronavirus, severe acute respiratory syndrome coronavirus 2 (SARS-CoV-2) ${ }^{1}$, has since been identified as the etiological agent, and the associated viral pneumonia has been named coronavirus disease 2019 (COVID-19)2.

This viral outbreak spread throughout China and to multiple other countries within 1 month of the first confirmed case ${ }^{3,4}$. On January 30, 2020, the World Health Organization (WHO) identified COVID-19 as a public health emergency of international concern ${ }^{5}$.

Multiple reports have described COVID-19 clinical symptoms like a dry cough, fever, dyspnea, and fatigue $4,6,7$. It has been reported that COVID-19 patients with underlying comorbidities (e.g., hypertension, diabetes mellitus, and cardiovascular disease) are at greater risk of severe acute respiratory syndrome (SARS) and death ${ }^{4}$. Because SARS-CoV-2 is a newly discovered virus, we have limited knowledge about the associated disease.

In the context of an ongoing outbreak, here we report the case of a 68-year-old man with no distinctive clinical feature, but with COVID-19 confirmed by real-time reverse transcriptase PCR (rRT-PCR), who had comorbidities and died of ruptured TAA within $19 \mathrm{~h}$ of hospital admission. We hope that by rapidly sharing this case report, we can draw attention to the potential lethality of COVID-19 plus TAA.

\section{Case Presentation}

At 09:00 on February 15, 2020, a 68-year-old man consulted an emergency physician in the First Affiliated Hospital of Zhengzhou University for a 10-day abdominalgia and a 6-day cough accompanied by objective fever (the highest axillary temperature was $39.1^{\circ} \mathrm{C}$ ). He presented with a mask and disclosed that he had come from Xinyang, Henan Province, China, which is the most severely COVID-19-affected city in Henan province. However, our patient did not admit having traveled to Hubei Province or having direct contact with the people who came from Hubei Provence and denied having any history of hypertension, diabetes mellitus, cardiovascular disease, tuberculosis or hypertriglyceridemia. He told the doctor that he had smoked for most of his life but had always been healthy. 
A physical examination found an axillary temperature of $38.5^{\circ} \mathrm{C}$, pulse of 106 beats per min, respiratory rate of 27 breaths per min, blood pressure of $159 / 85 \mathrm{mmHg}$, and oxygen saturation of $98 \%$ when the patient was receiving $3 \mathrm{~L} / \mathrm{min}$ of oxygen via nasal prongs; the oxygen saturation value dropped below $92 \%$ when he was breathing ambient air.

Considering that the patient had come from the most severely infected area in Henan Provence, nasopharyngeal swab specimens were collected as routine, and pathogens, including influenza $A$ and $B$, were tested for. Then, this patient was isolated in the emergency ward. Lung auscultation revealed diminished sounds and moist rales. The lab results were returned as negative for influenza $A$ and $B$ at 09:28, while nasopharyngeal swabs tested positive for COVID-19 by rRT-PCR at 16:40 the same day. The patient was immediately transferred to the isolation ward.

The patient complained of a 10-day history of consistent abdominal pain, which was accompanied by constipation. He did not report nausea and was not vomiting, but he could not clearly describe the precise position or character of the abdominal pain. He reported that he had malaise with breathing, and that he had sometimes coughed up white mucus during the past 4 days. Vital signs showed fever, tachypnoea, tachycardia, and raised blood pressure. At 19:27, the chest computed tomography (CT) and contrastenhanced CT detected bronchitis, emphysema, and thoracic aorta aneurysm (Figures 1, 2), but no particular abnormality was observed in the abdomen.

CT scan imaging identified diminished transparency of the lung and fuzzy margins, consistent with COVID-19, but no specific manifestation. As there is no specific treatment for COVID-19, therapy mainly consisted of symptom control. To deal with fever, the patient received $900 \mathrm{mg}$ of aspirin-lysine by intramuscular injection once. He also received $300 \mathrm{mg}$ doxofylline once by intravenous drip to treat his bronchitis and expectorant consisting of $90 \mathrm{mg}$ of ambroxol once by intravenous drip to manage his white mucus. Sodium nitroprusside $(25 \mathrm{mg}$ ) was pumped into the drip to control the high blood pressure. Lopinavir/ Ritonavir $400 \mathrm{mg} / 100 \mathrm{mg}$ film-coated tablets was given twice a day to help controlling virus. Blood tests, blood cell count, and serum chemical tests displayed mild erythropenia, leukocytosis, and thrombocytosis (Table 1). CT imaging indicated pneumonia, and clinical symptoms and blood counting suggested that bacterial pneumonia might also be present. Based on these findings, $400 \mathrm{mg}$ of moxifloxacin once per day and $3 \mathrm{~g}$ of sulperazon every $8 \mathrm{~h}$ were administered. Our patient was consistently supplied with oxygen by nasal cannula at $3 \mathrm{~L}$ per minute to maintain an oxygen saturation of around $98 \%$. The measurement of hepatic function revealed an albumin (Alb) level of $25.3 \mathrm{~g} / \mathrm{L}$, globulin (Glob) level of $43.4 \mathrm{~g} / \mathrm{L}$ and cholinesterase (Che) level of $3.00 \mathrm{KU} / \mathrm{L}$ (Table 1). At 23:21, the patient's procalcitonin (PCT) level increased to $1.340 \mathrm{ng} / \mathrm{mL}$ (reference range between $0-0.046 \mathrm{ng} / \mathrm{mL}$ ), C-Reactive Protein (CRP) level increased to $215.04 \mathrm{mg} / \mathrm{L}$ (reference range between $0-5 \mathrm{mg} / \mathrm{L}$ ), and interleukin 6 (IL-6) level increased to $156.80 \mathrm{pg} / \mathrm{mL}$ (reference range between $0-7 \mathrm{mg} / \mathrm{L}$ ).

The patient was under continuous electrocardiographic (ECG) monitoring after being admitted to the isolation ward. This patient disclosed that he felt better after supportive treatment given by his doctors. On February 16 at 03:10, the patient's condition rapidly worsened; the monitor alarmed that the blood 
pressure and heart rate of the patient could not be detected. The patient lost consciousness and had no response to his doctors' call. The patient had no bilateral pupillary light reflex and carotid artery pulse. In this

emergency, doctors placed the patient in a prostration position without a pillow and immediately performed cardiopulmonary resuscitation (CPR). Adrenaline ( $1 \mathrm{mg}$ ) was given every 3 minutes by intravenous injection, and $180 \mathrm{mg}$ dopamine was pumped in to increase heart rate and blood pressure. Intubation was operated by the doctors wearing appropriate protective equipment. An ultrasound technician and a vascular surgeon were invited for consultation. The chest ultrasound at our patient's bedside presented extensive pleural effusion, which did not show up by CT, and the doctors reached an agreement that the patient's aorta had ruptured. The patient's vital signs did not recover despite the rescue measures. A flat line was seen in the ECG monitor. At 04:12 on February 16, within $19 \mathrm{~h}$ of hospital admission, the patient was pronounced dead.

\section{Methods}

\section{Specimen collection and test}

Nasopharyngeal swab specimens were collected using synthetic fiber swabs by a nurse wearing appropriate protective equipment. The swabs were placed in a virus-collection tube containing $3.5 \mathrm{~mL}$ of transport medium. The collected specimens were tested immediately after collection by rRT-PCR. The whole specimen collection procedure followed the published guidelines ${ }^{8}$.

\section{Discussion}

\section{COVID-19 in our elderly patient}

The clinical manifestations of this 68-year-old man began abruptly: from the onset of abdominalgia to final death, the entire illness course was 10 days. The contrast-enhanced CT data were not suggestive of gastrointestinal disease. Given that there was no specific sign of gastrointestinal disease by CT scan imaging, it seems likely that our patient's abdominal discomfort was caused by COVID-19.

Four days after the onset of symptoms, he started to cough up white mucus. Moreover, the chest CT plain scan presented diminished transparency of the lung, fuzzy margins, fibrous stripes, and patchy shadows, with no clear ground-glass opacification. Blood counts were suggestive of leukocytosis, neutrophilia, and lymphopenia. The patient tested positive for coronavirus by rRT-PCR. These features suggest that this elderly man suffered COVID-19 combining with bacterial pneumonia.

Based on this clinical experience, COVID-19 diagnosis might be missed as bacterial pneumonia has more visible clinical features than COVID-19. Nevertheless, this case indicated that cytokine storm syndrome was the straw that broke the camel's back, resulting in the sudden death of our COVID-19 patient ${ }^{9}$. 


\section{COVID-19 and thoracic aorta aneurysm}

This patient's blood pressure was $159 / 85 \mathrm{mmHg}$ on admission, and chest contrast-enhanced CT found TAA. Hypertension is the leading cause of aortic aneurysm (AA). This patient died within $19 \mathrm{~h}$ of admission to the hospital, and the entire disease course lasted 10 days.

In this case, COVID-19 likely expediated an underlying AA, which likely resulted from a disbalance of angiotensin II (Ang II) and angiotensin-converting enzyme 2 (ACE2). Some reports have suggested that angiotensin II (Ang II) can induce AA ruptures ${ }^{10}$ and that the angiotensin-Converting Enzyme 2 (ACE2) inhibits Ang Il-induced abdominal AAs ${ }^{11}$. SARS-CoV-2 infects patients by binding to the ACE2 of humans, which leads to the exhaustion of ACE2 ${ }^{12}$. Given the rapid death of our COVID-19 patient with TAA, we hypothesize that COVID-19 might expedite the progress of TAA. Nevertheless, more convincing clinic and laboratory data are needed to confirm this hypothesis.

\section{COVID-19 and liver function damage}

The Alb (25.3 g/L) and Glob (43.4 g/L) results were suggestive of hepatic damage. The low Alb/Glob ratio $[(A / G)=0.583]$ also attracted our attention. In China, a low Alb/Glob ratio is typically caused by hepatitis $B$ virus infection, yet this patient was free of hepatitis $B$ and $C$. This finding is consistent with the notion that COVID-19 also attacks the liver, which is consistent with previous reports ${ }^{13,14}$.

\section{Conclusion}

Here we report the clinical features of COVID-19 in an elderly man, which were not distinctive, especially given that the patient had primary lung disease. COVID-19 patients with TAA should attract the highest vigilance as COVID-19 might expedite the process of TAA rupture, leading to rapid death with little or no warning signs. COVID-19 could progress rapidly to cause cytokine storm syndrome, worsening the prognosis.

Hepatic damage was observed in this COVID-19 patient who had no hepatic disease history. In response to this ongoing public health emergency, we have collected and summarized the data associated with this case, so that they can be shared with the international community. We would like to take this opportunity to kindly encourage all clinicians, scientists, and public health professionals to openly share clinical and research data in as timely a manner as possible, thereby helping to combat this viral threat.

\section{Declarations}

\section{Ethical Approval and Consent to participate}

We dealt with this case under the guideline of the Medical Ethics Committee of our affiliation for protecting patient privacy and confidentiality. We got consent from the son of our dead patient allowing 
us to report this case.

\section{Consent for publication}

Infectious Diseases of Poverty has our permission to publish this report. We got consent from the son of our dead patient allowing us to share this case by publishing.

\section{Availability of supporting data}

We would like to share all the clinic data about this case.

\section{Competing interests}

There are no financial conflicts of interest to report.

\section{Funding}

All authors received no specific funding for this work.

\section{Authors' contributions}

Kun Zhao wrote the manuscript. Zhang Cao revised the manuscript. Changju Zhu and Yi Zhang depicted the figures and tables. Feifan Chen collected all medical records of this report.

\section{Acknowledgments}

We thank the patient and his son, the nurses and clinical staff who are working at the Emergency department providing care for our patients, and all the members of the COVID-19 response teams of our hospital.

\section{Authors' information}

Kun Zhao, M.D, Zhang Cao, M.D., Changju Zhu, M.D., Yi Zhang, Ph.D., Feifan Chen, M.D*

Author Affiliations

All authors are from the First Affiliated Hospital of Zhengzhou University, Zhengzhou, Henan, China

*Corresponding author, Department of Emergency, Zhengzhou University First Affiliated Hospital, No.1 Eastern Jianshe Road, Zhengzhou, CN 450052, Email: chenfeifanzzu@gmail.com.

\section{References}

1. Gorbalenya AE. Severe acute respiratory syndrome-related coronavirus-The species and its viruses, a statement of the Coronavirus Study Group. BioRxiv 2020. 
2. Organization WH. Surveillance case definitions for human infection with novel coronavirus (nCoV). 2020.

3. Zhang Y, Xu J, Li H, Cao B. A novel coronavirus (COVID-19) outbreak: a call for action. Chest 2020.

4. Peeri NC, Shrestha N, Rahman MS, et al. The SARS, MERS and novel coronavirus (COVID-19) epidemics, the newest and biggest global health threats: what lessons have we learned? International Journal of Epidemiology 2020.

5. Organization WH. Statement on the second meeting of the International Health Regulations (2005) Emergency Committee regarding the outbreak of novel coronavirus (2019-nCoV). 2020.

6. Zhang JJ, Dong X, Cao YY, et al. Clinical characteristics of 140 patients infected by SARS-CoV-2 in Wuhan, China. Allergy 2020.

7. $X u X W, W u X X$, Jiang XG, et al. Clinical findings in a group of patients infected with the 2019 novel coronavirus (SARS-Cov-2) outside of Wuhan, China: retrospective case series. Bmj 2020;368:m606.

8. Control CfD, Prevention. Interim Guidelines for Collecting, Handling, and Testing Clinical Specimens from Persons Under Investigation (PUIs) for Coronavirus Disease 2019 (COVID-19). 2020.

9. Gong J, Dong H, Xia SQ, et al. Correlation Analysis Between Disease Severity and Inflammationrelated Parameters in Patients with COVID-19 Pneumonia. medRxiv 2020.

10. Stegbauer J, Thatcher SE, Yang G, et al. Mas receptor deficiency augments angiotensin Il-induced atherosclerosis and aortic aneurysm ruptures in hypercholesterolemic male mice. J Vasc Surg 2019;70:1658-68.e1.

11. Hao Q, Dong X, Chen X, et al. Angiotensin-Converting Enzyme 2 Inhibits Angiotensin II-Induced Abdominal Aortic Aneurysms in Mice. Hum Gene Ther 2018.

12. Sun ML, Yang JM, Sun YP, Su GH. Inhibitors of RAS Might Be a Good Choice for the Therapy of COVID-19 Pneumonia. Zhonghua Jie He He Hu Xi Za Zhi 2020;43:E014.

13. Liu C, Jiang ZC, Shao CX, et al. [Preliminary study of the relationship between novel coronavirus pneumonia and liver function damage: a multicenter study]. Zhonghua Gan Zang Bing Za Zhi 2020;28:148-52.

14. Zheng Y, Huang Z, Ying G, et al. Comparative study of the lymphocyte change between COVID-19 and non-COVID-19 pneumonia cases suggesting uncontrolled inflammation might not be the main reason of tissue injury. medRxiv 2020.

\section{Tables}

Due to technical limitations, Tables 1-2 are provided in the Supplementary Files section.

\section{Figures}



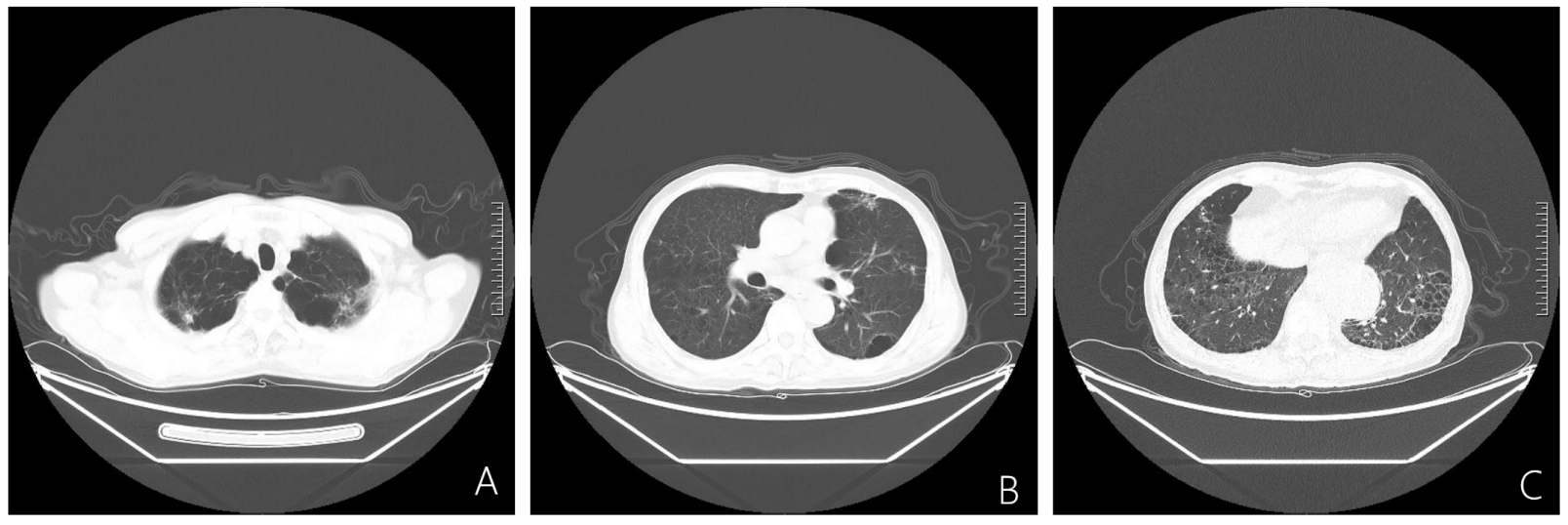

Figure 1. Three axial layers of chest computed tomography (CT), Feb 15, 2020.

\section{Figure 1}

Three axial layers of chest computed tomography 


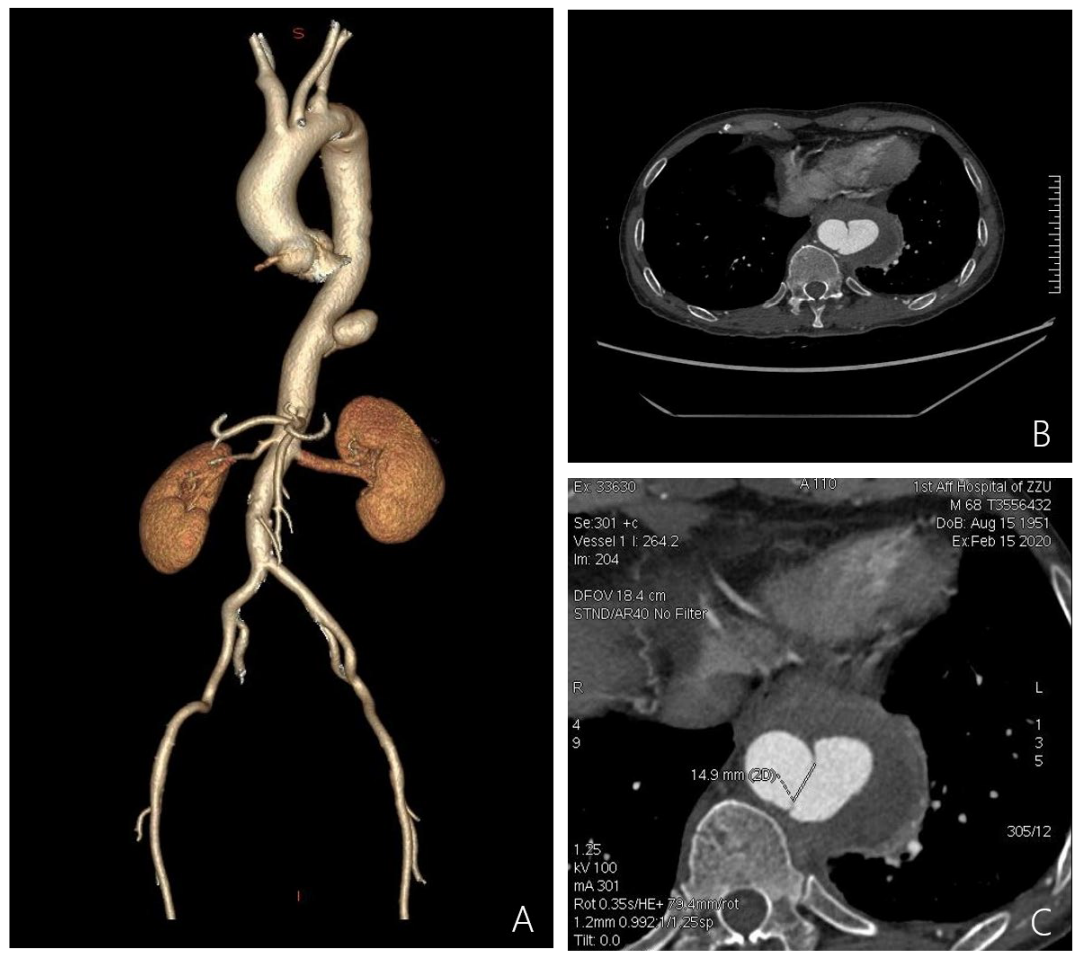

Figure 2. Images of Thoracic aortic aneurysm (TAA) in three-dimensional angiography (A) and contrast-enhanced CT (B, C), Feb 15, 2020.

\section{Figure 2}

Images of Thoracic aortic aneurysm (TAA) in three-dimensional angiography and contrast-enhanced CT

\section{Supplementary Files}

This is a list of supplementary files associated with this preprint. Click to download.

- table1.tif

- table2.tif 